\title{
The Signed Product Cordial of the Sum and Union of the Fourth Power of Paths with Cycles
}

\author{
Elrokh $\mathrm{A}^{1}$, Rabie $\mathrm{A}^{2 *}$ \\ ${ }^{\mathrm{T}}$ Dept of Math, Faculty of Science, Menoufia University, Egypt \\ ${ }^{2}$ Institute of National Planning,Cairo, Egypt \\ *Corresponding Author \\ Dr. Aya Rabie
}

\section{Article History}

Received: 19.02 .2020

Accepted: 26.02 .2020

Published: 08.03.2020

\begin{abstract}
In this paper, we contribute some new results on signed product cordial labeling and present necessary and sufficient conditions for signed product cordial in two cases:first ,the sum of the fourth power of paths with cycles and second, the union of the fourth power of paths with cycles.
\end{abstract}

Keywords: Fourth power, Sum graph, Union graph, Signed product cordial graph.

\section{INTRODUCTION}

Graph theory is well known subject in mathematics and computer science. Graph theory is now a major tool in mathematical research, marketing and so on. Graph labeling is one of the research area in graph theory. The concept of graph labeling was introduced by Rosa in 1967 [5]. A graph labeling is an assignment of integers to the vertices or edges or both subject to certain condition(s). Most of the graph labeling problems have the following three common characteristics: a set of numbers for assignment of vertex labels, a rule that assigns a label to each edge and some condition(s) that these labels must satisfy. For detailed survey on graph labeling we refer to A Dynamic Survey of Graph Labeling by Gallian [4]. Cordial labeling was introduced by Cahit [1] who called a graph $G$ cordial if there is a vertex labeling $f: V(G) \rightarrow\{0,1\}$ such that the induced labeling $f^{*}: E(G) \rightarrow\{0,1\}$, defined by $f^{*}(x y)=|f(x)-f(y)|$, for all edges $x, y \in E(G)$ and with the following inequalities holding: $\left|v_{f}(0)-v_{f}(1)\right| \leq 1$ and $\left|e_{f}(0)-e_{f}(1)\right| \leq 1$, where $v_{f}(i)$ (respectively $e_{f}(i)$ ) is the number of vertices (respectively, edges) labeled with $i$. Motivated through the concept of cordial labeling the product cordial labeling was introduced by Sundaram et al.[6] where absolute difference of vertex labels is replaced by product of vertex labels.

\section{Preliminaries}

In this section, we give the basic definitions relevant to this paper. Let $G(V, E)$ be a finite, simple and undirected graph with $p$ vertices and $q$ edges.

Definition 1. A vertex labeling of graph $G f: V(G) \rightarrow\{-1,1\}$ with induced edge labeling $f^{*}: E(G) \rightarrow\{-1,1\}$ defined by $f^{*}(u v)=f(u) \cdot f(v)$ is called a signed product cordial labeling if $\left|v_{f}(-1)-v_{f}(1)\right| \leq 1$ and $\left|e_{f}(-1)-e_{f}(1)\right| \leq 1$, where $v_{f}(-1)$ is the number of vertices labeled with $-1, v_{f}(1)$ is the number of vertices labeled 1 , e $\left(f^{*}\right)(-1)$ is the number of edges labeled with -1 and $\left.e_{(} f^{*}\right)(1)$ is the number of edges labeled 1 . A graph $G$ is signed product cordial if it admits signed product cordial labeling.

Definition 2. The fourth power of a cycles $C_{n}$, denoted by $C_{n}^{4}$, is $C_{n} \mathrm{U} J$, where $\mathrm{J}$ is the set of all edges of the form edges $v_{i} v_{j}$ such that $2 \leq d\left(v_{i} v_{j}\right) \leq 4$ and $i<j$ where $d\left(v_{i} v_{j}\right)$ is the shortest distance from $v_{i}$ to $v_{j}$.

Copyright @ 2020: This is an open-access article distributed under the terms of the Creative Commons Attribution license which permits unrestricted use, distribution, and reproduction in any medium for non commercial use (NonCommercial, or CC-BY-NC) provided the original author and source are credited. 
Definition 3. The Sum or join is $G_{1}+G_{2}$, is the graph with vertex set and edge set given by $V\left(G_{1}\right)+V\left(G_{2}\right)$ $=V\left(G_{1}\right) \cup V\left(G_{2}\right)$ and $E\left(G_{1}+G_{2}\right)=E\left(G_{1}\right) \cup E\left(G_{2}\right) \cup \quad J$, where $J$ consists of edges join each vertex of $G_{1}$ to every vertex of $G_{2}$.

Definition 4. The union is $G_{1} \cup \quad G_{2}=\left(\begin{array}{lll}V_{1} \cup \quad V_{2}, E_{1} \cup \quad E_{2}\end{array}\right)$ (simple graph). The fourth power of a cycles $C_{n}$ denoted by $C_{n}^{4}$ is $C_{n} \mathrm{U} J$, where $\mathrm{J}$ is the set of all edges of the form edges $v_{i} v_{j}$ such that $2 \leq d\left(v_{i} v_{j}\right) \leq 4$ and $i<j$.

Diab [2-3] has reported several results concerning the sum and union of the cycles $C_{n}$ and paths $P_{m}$ together and with other specific graphs.

\section{Terminologies and Notations}

A cycle with $n$ vertices and $n$ edges, denoted by $C_{n}$, and its fourth power $C_{n}^{4}$ has $n$ vertices and $4 n-9$ edges. We let $L_{4 r}$ denote the labeling $(-1)_{2} 11(-1)_{2} 11 \ldots(-1)_{2} 11$ (repeated $r$-times), Let $L_{4 r}^{\prime}$ denote the labeling $(-1) 11(-1)(-1) 11(-1) \ldots(-1) 11(-1)$ (repeated $r$-times).

The labeling $11(-1)_{2} 11(-1)_{2} \ldots 11(-1)_{2}$ (repeated $r$-times) and labeling $1(-1)_{2} 11(-1)_{2} 1 \ldots 1(-1)_{2} 1$ (repeated $r$-times) are written $S_{4 r}$ and $S_{4 r}^{\prime}$. Let $M_{r}$ denote the labeling $(-1) 1(-1) 1 \ldots(-1) 1$, zero-one repeated $r$ times if $r$ is even and $(-1) 1(-1) 1 \ldots(-1) 1(-1)$ if $r$ is odd; for example, $M_{6}=(-1) 1(-1) 1(-1) 1$ and $M_{5}=(-1) 1(-1) 1(-1)$. We let $M^{\prime}{ }_{r}$ denote the labeling $1(-1) 1(-1) \ldots 1(-1)$. Sometimes, we modify the labeling $M_{r}$ or $M^{\prime}{ }_{r}$ by adding symbols at one end or the other (or both). Also, $L_{4 r}$ (or $L^{\prime}{ }_{4 r}$ ) with extra labeling from right or left (or both sides).

If $L$ is a labeling for fourth power of paths $p_{m}$ and $M$ is a labeling for fourth power of paths $P_{n}$, then we use the notation $[L ; M]$ to represent the labeling of the sum $P_{m}^{4}+P_{n}^{4}$. Additional notation that we use is the following. For a given labeling of the sum $P_{m}^{4}+P_{n}^{4}$, we let $v_{i}$ and $e_{i}$ (for $i=-1,1$ ) be the numbers of labels that are $i$ as before, we let $x_{i}$ and $a_{i}$ be the corresponding quantities for $P_{m}^{4}$, and we let $y_{i}$ and $b_{i}$ be those for $P_{n}^{4}$. It is easy to verify that $v_{-1}-v_{1}=$ $\left(x_{-1}-x_{1}\right)+\left(y_{-1}-y_{1}\right)$ and $e_{-1}-e_{1}=\left(a_{-1}-a_{1}\right)+\left(b_{-1}-b_{1}\right)+\left(x_{-1}-x_{1}\right)\left(y_{-1}-y_{1}\right)$.

Also for $P_{m}^{4} \cup p_{n}^{4}$, we use the same notation $[L ; M]$. Additional notation that we use is the following. For a given labeling of the union $P_{m}^{4} \cup p_{n}^{4}$, we let $v_{i}$ and $e_{i}$ (for $i=-1,1$ ) be the numbers of labels that are $i$ as before, we let $x_{i}$ and $a_{i}$ be the corresponding quantities for $P_{m}^{4}$, and we let $y_{i}$ and $b_{i}$ be those for $p_{n}^{4}$. It is easy to verify that $v_{-1}-v_{1}=\left(x_{-1}-\right.$ $\left.x_{1}\right)+\left(y_{-1}-y_{1}\right)$ and $e_{-1}-e_{1}=\left(a_{-1}-a_{1}\right)+\left(b_{-1}-b_{1}\right)$. When it comes to the proof, we only need to show that, for each specified combination of labeling, $\left|v_{-1}-v_{1}\right| \leq 1$ and $\left|e_{-1}-e_{1}\right| \leq 1$.

\section{Main Results}

Signed product cordial of the sum of the fourth power of paths with cycles.

In this section, we study the signed product cordial of the sum of the fourth power of paths with cycles.

Lemma 4.1.1. If $n \equiv 0(\bmod 4)$, then $P_{n}^{4}+c_{m}^{4}$ is signed product cordial for all $n, m \geq 7$.

Proof. Suppose that $n=4 r$, where $r \geq 2$. The following cases will be examined.

Case $1 . m \equiv 0(\bmod 4)$.

Suppose that $m=4 s$, where $s \geq 2$. Then we label the vertices of $P_{4 r}^{4}+c_{4 s}^{4}$ by $\left[(-1) L_{4 r-4}(-1) 11 ; S_{4 s}^{\prime}\right]$. Therefore $x_{-1}=x_{1}=2 r, a_{-1}=a_{1}=8 r-5, y_{-1}=y_{1}=2 s, b_{-1}=8 s-4, b_{1}=8 s-5$. It follows that $v_{-1}-v_{1}=0$ and $e_{-1}-e_{1}=1$. As an example, Figure (1) illustrates $P_{8}^{4}+c_{8}^{4}$. Hence, $P_{4 r}^{4}+c_{4 s}^{4}$ is signed product cordial. 


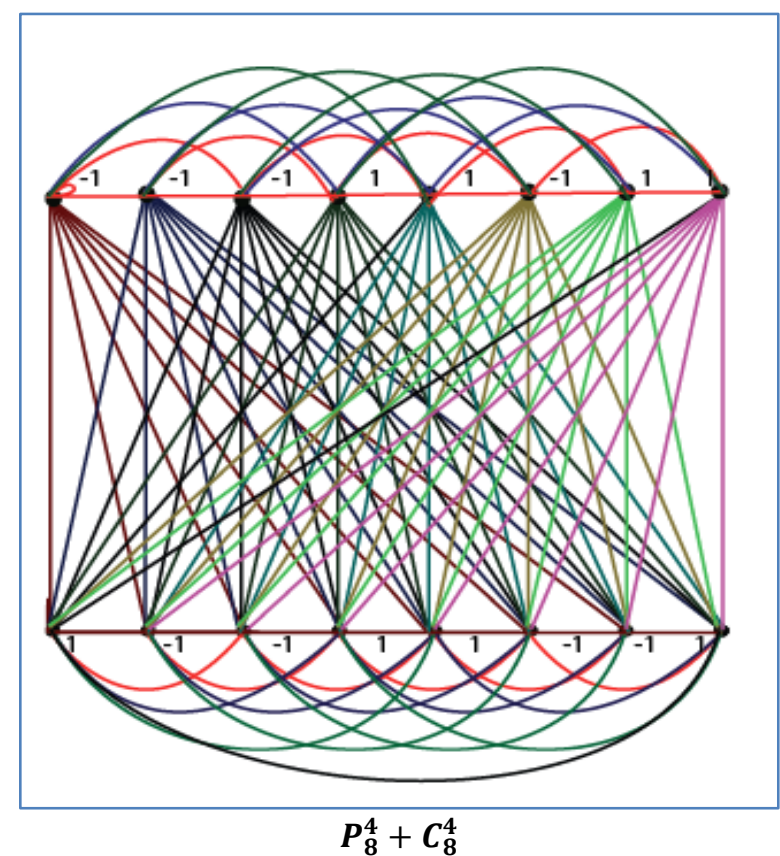

Fig-1

Case 2. $m \equiv 1(\bmod 4)$.

Suppose that $m=4 s+1$, where $s \geq 2$. Then we label the vertices of $P_{4 r}^{4}+c_{4 s+1}^{4}$ by $\left[(-1) L_{4 r-4}(-1) 11 ; L_{4 s}(-1)\right]$. Therefore $x_{-1}=x_{1}=2 r, a_{-1}=a_{1}=8 r-5, y_{-1}=2 s+1, y_{1}=2 s, b_{-1}=8 s-$ $2, b_{1}=8 s-3$. It follows that $v_{-1}-v_{1}=1$ and $e_{-1}-e_{1}=1$. Hence, $P_{4 r}^{4}+c_{4 s+1}^{4}$ is signed product cordial.

Case 3. $m \equiv 2(\bmod 4)$.

Suppose that $m=4 s+2$, where $s \geq 2$. Then we label the vertices of $P_{4 r}^{4}+c_{4 s+2}^{4}$ by $\left[(-1) L_{4 r-4}(-1) 11 ;(-1)_{3} 1_{3} L_{4 s-4}^{\prime}\right]$. Therefore $x_{-1}=x_{1}=2 r, a_{-1}=a_{1}=8 r-5, y_{-1}=y_{1}=2 s+1, b_{-1}=8 s, b_{1}=$ $8 s-1$. It follows that $v_{-1}-v_{1}=0$ and $e_{-1}-e_{1}=1$. Hence, $P_{4 r}^{4}+c_{4 s+2}^{4}$ is signed product cordial.

Case 4. $m \equiv 3(\bmod 4)$.

Suppose that $m=4 s+3$, where $s \geq 1$. Then we label the vertices of $P_{4 r}^{4}+c_{4 s+3}^{4}$ by $\left[(-1) L_{4 r-4}(-1) 11 ; L_{4 s}^{\prime}(-1) 1(-1)\right]$. Therefore $x_{-1}=x_{1}=2 r, a_{-1}=a_{1}=8 r-5, y_{-1}=2 s+2, y_{1}=2 s+1, b_{-1}=$ $8 s+2, b_{1}=8 s+1$. It follows that $v_{-1}-v_{1}=1$ and $e_{-1}-e_{1}=1$. Hence, $P_{4 r}^{4}+c_{4 s+3}^{4}$ is signed product cordial.

Lemma 4.1.2. If $n \equiv 1(\bmod 4)$, then $P_{n}^{4}+c_{m}^{4}$ is signed product cordial for all $n, m \geq 7$.

Proof. Suppose that $n=4 r+1$, where $r \geq 2$. The following cases will be examined.

Case 1. $m \equiv 0(\bmod 4)$.

Suppose that $m=4 s$, where $s \geq 2$. Then we label the vertices of $P_{4 r+1}^{4}+c_{4 s}^{4}$ by $\left[(-1)_{2} L_{4 r-4} 1(-1) 1 ; S^{\prime}{ }_{4 s}\right]$. Therefore $\quad x_{-1}=2 r+1, x_{1}=2 r, a_{-1}=a_{1}=8 r-3, y_{-1}=y_{1}=2 s, b_{-1}=8 s-4, b_{1}=8 s-5$. It follows that $v_{-1}-v_{1}=1$ and $e_{-1}-e_{1}=1$. Hence, $P_{4 r+1}^{4}+c_{4 s}^{4}$ is signed product cordial.

Case 2. $m \equiv 1(\bmod 4)$.

Suppose that $m=4 s+1$, where $s \geq 2$. Then we label the vertices of $P_{4 r+1}^{4}+c_{4 s+1}^{4}$ by $\left[(-1)_{2} L_{4 r-4} 1(-1) 1 ; 1_{3} L_{4 s-4}^{\prime}(-1)_{2}\right]$. Therefore $x_{-1}=2 r+1, x_{1}=2 r, a_{-1}=a_{1}=8 r-3, y_{-1}=2 s, y_{1}=2 s+$ $1, b_{-1}=8 s-3, b_{1}=8 s-2$. It follows that $v_{-1}-v_{1}=0$ and $e_{-1}-e_{1}=0$. Hence, $P_{4 r+1}^{4}+c_{4 s+1}^{4}$ is signed product cordial.

\section{Case 3. $m=2(\bmod 4)$}

Suppose that $m=4 s+2$, where $s \geq 2$. Then we label the vertices of $P_{4 r+1}^{4}+c_{4 s+2}^{4}$ by $\left[(-1)_{2} L_{4 r-4} 1(-1) 1 ;(-1)_{3} 1_{3} L_{4 s-4}^{\prime}\right.$. Therefore $x_{-1}=2 r+1, x_{1}=2 r, a_{-1}=a_{1}=8 r-3, y_{-1}=y_{1}=2 s+1, b_{-1}=$ $8 s, b_{1}=8 s-1$. It follows that $v_{-1}-v_{1}=1$ and $e_{-1}-e_{1}=1$. Hence, $P_{4 r+1}^{4}+c_{4 s+2}^{4}$ is signed product cordial. 
Case 4. $m=3(\bmod 4)$.

Suppose that $m=4 s+3$, where $s \geq 2$. Then we label the vertices of $P_{4 r+1}^{4}+c_{4 s+3}^{4}$ by $\left[1_{2} L_{4 r-4}^{\prime}(-1) 1(-1) ;(-1) 11(-1) S_{4 s-4} 1(-1)_{2}\right]$. Therefore $x_{-1}=2 r, x_{1}=2 r+1, a_{-1}=a_{1}=8 r-3, y_{-1}=2 s+$ $2, y_{1}=2 s+1, b_{-1}=8 s+1, b_{1}=8 s+2$. It follows that $v_{-1}-v_{1}=0$ and $e_{-1}-e_{1}=0$. Hence, $P_{4 r+1}^{4}+c_{4 s+3}^{4}$ is signed product cordial.

Lemma 4.1.3. If $n \equiv 2(\bmod 4)$, then $P_{n}^{4}+c_{m}^{4}$ is Signed-signed product cordial for all $m, n \geq 7$.

Proof. Suppose that $n=4 r+2$, where $r \geq 2$. The following cases will be examined.

Case $1 . m \equiv 0(\bmod 4)$.

Suppose that $m=4 s$, where $s \geq 2$. Then we label the vertices of $P_{4 r+2}^{4}+c_{4 s}^{4}$ by $\left[(-1) 1_{3}(-1) S_{4 r-4}(-1) ; S^{\prime}{ }_{4 s}\right]$. Therefore $x_{-1}=x_{1}=2 r+1, a_{-1}=a_{1}=8 r-1, y_{-1}=y_{1}=2 s, b_{-1}=8 s-4, b_{1}=8 s-5$. It follows that $v_{-1}-$ $v_{1}=0$ and $e_{-1}-e_{1}=1$. Hence, $P_{4 r+2}^{4}+c_{4 s}^{4}$ is signed product cordial.

Case 2. $m \equiv 1(\bmod 4)$.

Suppose that $m=4 s+1$, where $s \geq 2$. Then we label the vertices of $P_{4 r+2}^{4}+c_{4 s+1}^{4}$ by $\left[(-1) 1_{3}(-1) S_{4 r-4}(-1) ; L_{4 s}(-1)\right]$. Therefore $x_{-1}=x_{1}=2 r+1, a_{-1}=a_{1}=8 r-1, y_{-1}=2 s+1, y_{1}=2 s, b_{-1}=$ $8 s-2, b_{1}=8 s-3$. It follows that $v_{-1}-v_{1}=1$ and $e_{-1}-e_{1}=1$. Hence, $P_{4 r+2}^{4}+c_{4 s+1}^{4}$ is signed product cordial.

Case 3. $m \equiv 2(\bmod 4)$.

Suppose that $m=4 s+2$, where $s \geq 2$. Then we label the vertices of $P_{4 r+2}^{4}+c_{4 s+2}^{4}$ by $\left[(-1) 1_{3}(-1) S_{4 r-4}(-1) ;(-1)_{3} 1_{3} L_{4 s-4}^{\prime}\right]$. Therefore $x_{-1}=x_{1}=2 r+1, a_{-1}=a_{1}=8 r-1, y_{-1}=y_{1}=2 s+1, b_{-1}=$ $8 s, b_{1}=8 s-1$. It follows that $v_{-1}-v_{1}=0$ and $e_{-1}-e_{1}=1$. Hence, $P_{4 r+2}^{4}+c_{4 s+2}^{4}$ is signed product cordial.

Case 4. $m \equiv 3(\bmod 4)$.

Suppose that $m=4 s+3$, where $s \geq 1$. Then we label the vertices of $P_{4 r+2}^{4}+c_{4 s+3}^{4}$ by $\left[(-1) 1_{3}(-1) S_{4 r-4}(-1) ; L_{4 s}^{\prime}(-1) 1(-1)\right]$. Therefore $x_{-1}=x_{1}=2 r+1, a_{-1}=a_{1}=8 r-1, y_{-1}=2 s+2, y_{1}=2 s+$ $1, b_{-1}=8 s+2, b_{1}=8 s+1$. It follows that $v_{-1}-v_{1}=1$ and $e_{-1}-e_{1}=1$. Hence, $P_{4 r+2}^{4}+c_{4 s+3}^{4}$ is signed product cordial.

Lemma 4.1.4. If $n \equiv 3(\bmod 4)$, then $P_{n}^{4}+c_{m}^{4}$ is Signed product cordial for all $m, n \geq 7$.

Proof. Suppose that $n=4 r+3$, where $r \geq 2$. The following cases will be examined.

Case $1 . m \equiv 0(\bmod 4)$.

Suppose that $m=4 s$, where $s \geq 2$. Then we label the vertices of $P_{4 r+3}^{4}+c_{4 s}^{4}$ by $\left[(-1)_{2} 1 L_{4 r} ; S^{\prime}{ }_{4 s}\right]$. Therefore $x_{-1}=2 r+2, x_{1}=2 r+1, a_{-1}=a_{1}=8 r+1, y_{-1}=y_{1}=2 s, b_{-1}=8 s-4, b_{1}=8 s-5$. It follows that $v_{-1}-v_{1}=$ 1 and $e_{-1}-e_{1}=1$. Hence, $P_{4 r+3}^{4}+c_{4 s}^{4}$ is signed product cordial.

Case $2 . m \equiv 1(\bmod 4)$.

Suppose that $m=4 s+1$, where $s \geq 2$. Then we label the vertices of $P_{4 r+3}^{4}+c_{4 s+1}^{4}$ by $\left[(-1)_{2} 1 L_{4 r} ; 1_{3} L_{4 s-4}^{\prime}(-1)_{2}\right]$. Therefore $x_{-1}=2 r+2, x_{1}=2 r+1, a_{-1}=a_{1}=8 r+1, y_{-1}=2 s, y_{1}=2 s+1, b_{-1}=$ $8 s-3, b_{1}=8 s-2$. It follows that $v_{-1}-v_{1}=0$ and $e_{-1}-e_{1}=0$. Hence, $P_{4 r+3}^{4}+c_{4 s+1}^{4}$ is signed product cordial.

Case 3. $m \equiv 2(\bmod 4)$.

Suppose that $m=4 s+2$, where $s \geq 2$. Then we label the vertices of $P_{4 r+3}^{4}+c_{4 s+2}^{4}$ by $\left[(-1)_{2} 1 L_{4 r} ;(-1)_{3} 1_{3} L_{4 s-4}^{\prime}\right]$. Therefore $x_{-1}=2 r+2, x_{1}=2 r+1, a_{-1}=a_{1}=8 r+1, y_{-1}=y_{1}=2 s+1, b_{-1}=$ $8 s, b_{1}=8 s-1$. It follows that $v_{-1}-v_{1}=1$ and $e_{-1}-e_{1}=1$. Hence, $P_{4 r+3}^{4}+c_{4 s+2}^{4}$ is signed product cordial.

Case 4. $m \equiv 3(\bmod 4)$.

Suppose that $m=4 s+3$, where $s \geq 2$. Then we label the vertices of $P_{4 r+3}^{4}+c_{4 s+3}^{4}$ by $\left[1_{2} S_{4 r}(-1) ;(-1) 1_{2}(-1) S_{4 s-4} 1(-1)_{2}\right]$. Therefore $x_{-1}=2 r+1, x_{1}=2 r+2, a_{-1}=a_{1}=8 r+1, y_{-1}=2 s+2, y_{1}=$ $2 s+1, b_{-1}=8 s+2, b_{1}=8 s+1$. It follows that $v_{-1}-v_{1}=0$ and $e_{-1}-e_{1}=0$. Hence, $P_{4 r+3}^{4}+c_{4 s+3}^{4}$ is signed product cordial.

As a consequence of all lemmas mentioned above we conclude the following theorem.

Theorem 4.1 $P_{n}^{4}+c_{m}^{4}$ is signed product cordial for all $n$ and all $m$ except $n, m$ both equal to 7 . 


\section{Signed product cordial of the union of the fourth power of paths with cycles}

In this section, we study the signed product cordial of the union of the fourth power of paths with cycles.

Lemma 4.2.1. If $n \equiv 0(\bmod 4)$, then $P_{n}^{4} \cup c_{m}^{4}$ is Signed product cordial for all $m, n \geq 7$.

Proof. Suppose that $n=4 r$, where $r \geq 2$. The following cases will be examined.

Case $1 . m \equiv 0(\bmod 4)$.

Suppose that $m=4 s$, where $s \geq 2$. Then we label the vertices of $P_{4 r}^{4} \cup c_{4 s}^{4}$ by $\left[(-1) L_{4 r-4}(-1) 11 ; S^{\prime}{ }_{4 s}\right]$. Therefore $x_{-1}=x_{1}=2 r, a_{-1}=a_{1}=8 r-5, y_{-1}=y_{1}=2 s, b_{-1}=8 s-4, b_{1}=8 s-5$. It follows that $v_{-1}-v_{1}=0$ and $e_{-1}-e_{1}=-1$. As an example, Figure (2) illustrates $P_{8}^{4} \cup c_{8}^{4}$. Hence, $P_{4 r}^{4} \cup c_{4 s}^{4}$ is signed product cordial.

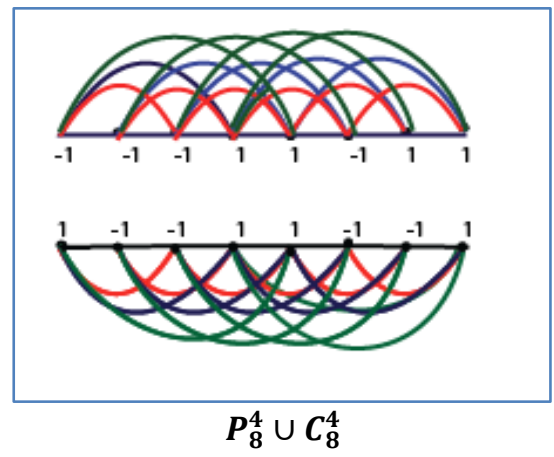

Fig-2

Case 2. $m \equiv 1(\bmod 4)$.

Suppose that $m=4 s+1$, where $s \geq 2$. Then we label the vertices of $P_{4 r}^{4} \cup c_{4 s+1}^{4}$ by $\left[(-1) L_{4 r-4}(-1) 11 ; L_{4 s}(-1)\right]$. Therefore $x_{-1}=x_{1}=2 r, a_{-1}=a_{1}=8 r-5, y_{-1}=2 s, y_{1}=2 s+1, b_{-1}=8 s-$ $2, b_{1}=8 s-3$. It follows that $v_{-1}-v_{1}=1$ and $e_{-1}-e_{1}=1$. Hence, $P_{4 r}^{4} \cup c_{4 s+1}^{4}$ is signed product cordial.

Case 3. $m \equiv 2(\bmod 4)$.

Suppose that $m=4 s+2$, where $s \geq 2$. Then we label the vertices of $P_{4 r}^{4} \cup c_{4 s+2}^{4}$ by $\left[(-1) L_{4 r-4}(-1) 11 ;(-1)_{3} 1_{3} L_{4 s-4}^{\prime}\right]$. Therefore $x_{-1}=x_{1}=2 r, a_{-1}=a_{1}=8 r-5, y_{-1}=y_{1}=2 s+1, b_{-1}=8 s, b_{1}=$ $8 s-1$. It follows that $v_{-1}-v_{1}=0$ and $e_{-1}-e_{1}=1$. Hence, $P_{4 r}^{4} \cup c_{4 s+2}^{4}$ is signed product cordial.

Case 4. $m \equiv 3(\bmod 4)$.

Suppose that $m=4 s+3$, where $s \geq 1$. Then we label the vertices of $P_{4 r}^{4} \cup c_{4 s+3}^{4}$ by $\left[(-1) L_{4 r-4}(-1) 11 ; L_{4 s}^{\prime}(-1) 1(-1)\right]$. Therefore $x_{-1}=x_{1}=2 r, a_{-1}=a_{1}=8 r-5, y_{-1}=2 s+2, y_{1}=2 s+1, b_{-1}=$ $8 s+2, b_{1}=8 s+1$. It follows that $v_{-1}-v_{1}=1$ and $e_{-1}-e_{1}=1$. Hence, $P_{4 r}^{4} \cup c_{4 s+3}^{4}$ is signed product cordial.

Lemma 4.2.2. If $n \equiv 1(\bmod 4)$, then $P_{n}^{4} \cup c_{m}^{4}$ is Signed product cordial for all $m, n \geq 7$.

Proof. Suppose that $n=4 r+1$, where $r \geq 2$. The following cases will be examined.

Case 1. $m \equiv 0(\bmod 4)$.

Suppose that $m=4 s$, where $s \geq 2$. Then we label the vertices of $P_{4 r+1}^{4} \cup c_{4 s}^{4}$ by $\left[(-1)_{2} L_{4 r-4} 1(-1) 1 ; S^{\prime}{ }_{4 s}\right]$. Therefore $\quad x_{-1}=2 r+1, x_{1}=2 r, a_{-1}=a_{1}=8 r-3, y_{-1}=y_{1}=2 s, b_{-1}=8 s-4, b_{1}=8 s-5$. It follows that $v_{-1}-v_{1}=1$ and $e_{-1}-e_{1}=1$. Hence, $P_{4 r+1}^{4} \cup c_{4 s}^{4}$ is signed product cordial.

Case $2 . m \equiv 1(\bmod 4)$.

Suppose that $m=4 s+1$, where $s \geq 2$. Then we label the vertices of $P_{4 r+1}^{4} \cup c_{4 s+1}^{4}$ by $\left[(-1)_{2} L_{4 r-4} 1(-1) 1 ; 1_{3} L_{4 s-4}^{\prime}(-1)(-1)\right]$. Therefore $x_{-1}=2 r+1, x_{1}=2 r, a_{-1}=a_{1}=8 r-3, y_{-1}=2 s, y_{1}=2 s+$ $1, b_{-1}=8 s-3, b_{1}=8 s-2$. It follows that $v_{-1}-v_{1}=0$ and $e_{-1}-e_{1}=-1$. Hence, $P_{4 r+1}^{4} \cup c_{4 s+1}^{4}$ is signed product cordial.

Case 3. $m \equiv 2(\bmod 4)$.

Suppose that $m=4 s+2$, where $s \geq 2$. Then we label the vertices of $P_{4 r+1}^{4} \cup c_{4 s+2}^{4}$ by $\left[(-1)_{2} L_{4 r-4} 1(-1) 1 ;(-1)_{3} 1_{3} L_{4 s-4}^{\prime}\right]$. Therefore $x_{-1}=2 r+1, x_{1}=2 r, a_{-1}=a_{1}=8 r-3, y_{-1}=y_{1}=2 s+1, b_{-1}=$ $8 s, b_{1}=8 s-1$. It follows that $v_{-1}-v_{1}=1$ and $e_{-1}-e_{1}=1$. Hence, $P_{4 r+1}^{4} \cup c_{4 s+2}^{4}$ is signed product cordial. 
Case 4. $m \equiv 3(\bmod 4)$.

Suppose that $m=4 s+3$, where $s \geq 1$. Then we label the vertices of $P_{4 r+1}^{4} \cup c_{4 s+3}^{4}$ by $\left[1_{2} L_{4 r-4}^{\prime}(-1) 1(-1) ; L_{4 s}^{\prime}(-1) 1(-1)\right]$. Therefore $x_{-1}=2 r, x_{1}=2 r+1, a_{-1}=a_{1}=8 r-3, y_{-1}=2 s+2, y_{1}=2 s+$ $1, b_{-1}=8 s+2, b_{1}=8 s+1$. It follows that $v_{-1}-v_{1}=0$ and $e_{-1}-e_{1}=1$. Hence, $P_{4 r+1}^{4} \cup c_{4 s+3}^{4}$ is signed product cordial.

Lemma 4.2.3. If $n \equiv 2(\bmod 4)$, then $P_{n}^{4} \cup c_{m}^{4}$ is Signed product cordial for all $m, n \geq 7$.

Proof. Suppose that $n=4 r+2$, where $r \geq 2$. The following cases will be examined.

Case $1 . m \equiv 0(\bmod 4)$.

Suppose that $m=4 s$, where $s \geq 2$. Then we label the vertices of $P_{4 r+2}^{4} \cup c_{4 s}^{4}$ by $\left[(-1) 1_{3}(-1) S_{4 r-4}(-1) ; S^{\prime}{ }_{4 s}\right]$. Therefore $x_{-1}=x_{1}=2 r+1, a_{-1}=a_{1}=8 r-1, y_{-1}=y_{1}=2 s, b_{-1}=8 s-4, b_{1}=8 s-5$. It follows that $v_{-1}-$ $v_{1}=0$ and $e_{-1}-e_{1}=1$. Hence, $P_{4 r+2}^{4} \cup c_{4 s}^{4}$ is signed product cordial.

Case 2. $m \equiv 1(\bmod 4)$.

Suppose that $m=4 s+1$, where $s \geq 2$. Then we label the vertices of $P_{4 r+2}^{4} \cup c_{4 s+1}^{4}$ by $\left[(-1) 1_{3}(-1) S_{4 r-4}(-1) ; L_{4 s}(-1)\right]$. Therefore $x_{-1}=x_{1}=2 r+1, a_{-1}=a_{1}=8 r-1, y_{-1}=2 s+1, y_{1}=2 s, b_{-1}=$ $8 s-2, b_{1}=8 S-3$. It follows that $v_{-1}-v_{1}=1$ and $e_{-1}-e_{1}=1$. Hence, $P_{4 r+2}^{4} \cup c_{4 S+1}^{4}$ is signed product cordial.

Case 3. $m \equiv 2(\bmod 4)$.

Suppose that $m=4 S+2$, where $S \geq 2$. Then we label the vertices of $P_{4 r+2}^{4} \cup c_{4 S+2}^{4}$ by $\left[(-1) 1_{3}(-1) S_{4 r-4}(-1) ;(-1)_{3} 1_{3} L_{4 S-4}^{\prime}\right]$. Therefore $\quad x_{-1}=x_{1}=2 r+1, a_{-1}=a_{1}=8 r-1, y_{-1}=y_{1}=2 S+$ $1, b_{-1}=8 S, b_{1}=8 S-1$. It follows that $v_{-1}-v_{1}=0$ and $e_{-1}-e_{1}=1$. Hence, $P_{4 r+2}^{4} \cup c_{4 S+2}^{4}$ is signed product cordial.

Case 4. $m \equiv 3(\bmod 4)$.

Suppose that $m=4 S+3$, where $S \geq 1$. Then we label the vertices of $P_{4 r+2}^{4} \cup c_{4 S+3}^{4}$ by $\left[(-1) 1_{3}(-1) S_{4 r-4}(-1) ; L_{4 S}^{\prime}(-1) 1(-1)\right]$. Therefore $x_{-1}=x_{1}=2 r+1, a_{-1}=a_{1}=8 r-1, y_{-1}=2 S+2, y_{1}=2 S+$ $1, b_{-1}=8 S+2, b_{1}=8 S+1$. It follows that $v_{-1}-v_{1}=1$ and $e_{-1}-e_{1}=1$. Hence, $P_{4 r+2}^{4} \cup c_{4 S+3}^{4}$ is signed product cordial.

Lemma 4.2.4. If $n \equiv 3(\bmod 4)$, then $P_{n}^{4} \cup c_{m}^{4}$ is Signed product cordial for all $m, n \geq 7$.

Proof. Suppose that $n=4 r+3$, where $r \geq 2$. The following cases will be examined.

Case $1 . m \equiv 0(\bmod 4)$.

Suppose that $m=4 S$, where $S \geq 2$. Then we label the vertices of $P_{4 r+3}^{4} \cup c_{4 S}^{4}$ by $\left[(-1)_{2} 1 L_{4 r} ; S^{\prime}{ }_{4 S}\right]$. Therefore $x_{-1}=2 r+2, x_{1}=2 r+1, a_{-1}=a_{1}=8 r+1, y_{-1}=y_{1}=2 S, b_{-1}=8 S-4, b_{1}=8 S-5$. It follows that $v_{-1}-v_{1}=$ 1 and $e_{-1}-e_{1}=1$. Hence, $P_{4 r+3}^{4} \cup c_{4 S}^{4}$ is signed product cordial.

Case $2 . m \equiv 1(\bmod 4)$.

Suppose that $m=4 S+1$, where $S \geq 2$. Then we label the vertices of $P_{4 r+3}^{4} \cup c_{4 S+1}^{4}$ by $\left[(-1)_{2} 1 L_{4 r} ; 1_{3} L_{4 S-4}^{\prime}(-1)_{2}\right]$. Therefore $x_{-1}=2 r+2, x_{1}=2 r+1, a_{-1}=a_{1}=8 r+1, y_{-1}=2 S, y_{1}=2 S+1, b_{-1}=$ $8 S-3, b_{1}=8 S-2$. It follows that $v_{-1}-v_{1}=0$ and $e_{-1}-e_{1}=-1$. Hence, $P_{4 r+3}^{4} \cup c_{4 S+1}^{4}$ is signed product cordial.

Case 3. $m \equiv 2(\bmod 4)$.

Suppose that $m=4 S+2$, where $S \geq 2$. Then we label the vertices of $P_{4 r+3}^{4} \cup c_{4 S+2}^{4}$ by $\left[(-1)_{2} 1 L_{4 r} ;(-1)_{3} 1_{3} L_{4 S-4}^{\prime}\right]$. Therefore $x_{-1}=2 r+2, x_{1}=2 r+1, a_{-1}=a_{1}=8 r+1, y_{-1}=y_{1}=2 S+1, b_{-1}=$ $8 S, b_{1}=8 S-1$. It follows that $v_{-1}-v_{1}=1$ and $e_{-1}-e_{1}=1$. Hence, $P_{4 r+3}^{4} \cup c_{4 S+2}^{4}$ is signed product cordial.

Case 4. $m \equiv 3(\bmod 4)$.

Suppose that $m=4 S+3$, where $S \geq 1$. Then we label the vertices of $P_{4 r+3}^{4} \cup c_{4 S+3}^{4}$ by $\left[1_{2} S_{4 r}(-1) ; L_{4 S}^{\prime}(-1) 1(-1)\right]$. Therefore $x_{-1}=2 r+1, x_{1}=2 r+2, a_{-1}=a_{1}=8 r+1, y_{-1}=2 S+2, y_{1}=2 S+$ $1, b_{-1}=8 S+2, b_{1}=8 S+1$. It follows that $v_{-1}-v_{1}=0$ and $e_{-1}-e_{1}=1$. Hence, $P_{4 r+3}^{4} \cup c_{4 S+3}^{4}$ is signed product cordial. 
As a consequence of all lemmas mentioned above we conclude the following theorem.

Theorem 4.1 $P_{n}^{4} \cup c_{m}^{4}$ is signed product cordial for all $n$ and all $m$.

\section{Conclusion}

In this paper we test the signed product cordial of the Sum and Union of fourth power of paths with cycles, we found that $P_{n}^{4} \cup c_{m}^{4}$ is cordial for all $m, n \geq 7$ and $P_{n}^{4}+c_{m}^{4}$ is cordial for all $m, n$ except $m, n$ both equal to 7 .

\section{ACKNOWLEDGEMENT}

The authors are thankful to the anonymous referee for useful suggestions and valuable comments.

\section{REFERENCES}

1. Cahit, C.G.(1987). A weaker version of graceful and harmonious Graphs, Ars Combinatoria, 23:201-207.

2. Diab, A .T.(2009). Study of some problems of cordial graphs, Ars Combin, 92, 255-261.

3. Diab, A.T.(2011). Generalization of some results on cordial graphs, Ars Combin, 99, 161-173.

4. Gallian, J. A.(2014). A Dynamic Survey of Graph Labeling, The Electronic Journal of Combinatorics, DS6.

5. Rosa, A.(1967). On certain valuations of the vertices of a graph, Theory of graphs (Internat. Symposium, Rome, July 1996), Gordon and Breach, N. Y. and Dunod Paris. 349, 355

6. Sundaram, M., Ponraj, R., \& Somasundaram, S.(2004). Product cordial labeling of graph, Bulletin of Pure and Applied Science, 23, 155-163. 